\title{
Nicht-Interventionelle Studien (NIS) in Österreich
}

Nicht-interventionelle Studien (NIS) oder Anwendungsbeobachtungen (AWB) evaluieren Arzneimittel und Medizinprodukte nach der Marktzulassung für die zugelassene Verwendung beobachtend in der Routineversorgung. Ob es sich hierbei um wertvolle Erkenntnisquellen oder lediglich um ein Marketinginstrument handelt, wird seit Jahren kontrovers diskutiert. In Österreich sind NIS nicht genehmigungs-, aber meldepflichtig. Die NIS müssen in einer Studiendatenbank (Register) registriert werden, in der einige Informationen auch öffentlich zugänglich gemacht werden. Das AIHTA ging erneut den Fragen nach, wieviele NIS in Österreich in welchem Umfang laufen, wer sie verantwortet und worin ihr Erkenntnisinteresse liegt. Dazu wurden die im Juli 2021 in der teil-öffentlichen Datenbank des BASG/AGES verfügbaren Informationen inklusive der veröffentlichten Kurzfassungen zu den Abschlussberichten ausgewertet. Wie schon in den Analysen zuvor wurden dem Register formale Mängel attestiert, die dazu führen, dass das Register in seiner derzeitigen Form keinen Beitrag zur Unterstützung der kritischen Öffentlichkeit leistet.

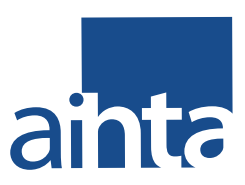

HTA Austria

Austrian Institute for Health Technology Assessment $\mathrm{GmbH}$

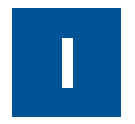
m BASG/AGES NIS-Register waren zum Stichtag 462 NIS registriert. Im Vergleich zum Erstbericht aus 2016 stieg die Gesamtanzahl der NIS um $84 \%$ (2016: 251 NIS) sowie die Patienten- und Patientinnenanzahl um 86\% (2021: 757.948, 2016: $406.831 \mathrm{~Pa}-$ tienten). Die Urheber der eingespeisten NIS sind weiterhin überwiegend Pharmafirmen. So stammten 292 der 462 NIS (d.h. 63\%) von diesen Organisationen im Erstbericht betrug dieser Anteil noch $76 \%$. Auf universitäre Einrichtungen entfielen 34 NIS (2016/2017: 37), während andere (Auftrags-)Forschungseinrichtungen mit 128 NIS (Anteil: 28\%, 2016/2017: 23) im Register vertreten waren. Bei der Mehrzahl der NIS handelt es sich um Anwendungsbeobachtungen, die Fragen zur Wirksamkeit unter Alltagsbedingungen, Verträglichkeit und Praktikabilität sowie zur langfristigen Sicherheit der Studienmedikamentierung erkundeten. Eine der größten Hürden in der Auswertung bestand in der Beschaffenheit des Regis- ters selbst: So bemängelte das AIHTA vor allem, dass Titel und Forschungsfragen erst in den Abschlussberichten konkretisiert werden, Kategorisierungen widersprüchlich erfolgen und/oder nicht mit den hochgeladenen Ergebnissen abgeglichen werden. Es dürfte daher vom Register-Betreiber BASG/AGES keine oder nur eine sehr oberflächliche, formale Kontrolle der eingereichten Datensätze erfolgen. Zu diesem Schluss kam das AIHTA bereits im Erstbericht bzw. Update.

Die deutsche Kassenärztliche Bundesvereinigung (KBV) listete 2020 vier Aspekte auf, wonach AWB kritisch zu bewerten sind. Drei dieser Aspekte (ungewöhnlich hohe Teilnehmerzahlen, Studien zu längst eingeführten und gut erprobten Arzneimitteln, mehrere unterschiedliche/ einander sehr ähnliche AWB zum selben Arzneimittel) ließen sich aus dem österreichischen NIS-Register ableiten. Die Arzneimittelkommission der deutschen Ärzteschaft (AkdÄ) rät inzwischen expli- zit von der Teilnahme an Anwendungsbeobachtungen ab. Die Gründe: geringer Erkenntnisgewinn und forcierter Einsatz teurer Medikamente bei existierenden Alternativen, um Marktanteile zu gewinnen. Eine 2015 in Deutschland durchgeführte Analyse zu Anwendungsbeobachtungen stellte zudem fest, dass jeder zehnte niedergelassene Arzt an AWB teilnimmt und infolge Studienmedikamente 7-8\% häufiger verschreibt als seine Kolleginnen und Kollegen.

\section{Originalpublikation:}

Sehic 0., Wild C. Nicht-Interventionelle Studien (NIS) in Österreich, 2. Update der systematischen Analysen 2016 \& 2017. AlHTA Policy Brief Nr.: 010 2021. Wien: HTA Austria - Austrian Institute for Health Technology Assessment GmbH. https://eprints.aihta.at/1333/.

\section{Kontakt:}

Ozren Sehic, B.A.

ozren.sehic@aihta.at

http://www.aihta.at 